Polymer Journal, Vol. 9, No. 3, pp 239-251 (1977)

\title{
Distribution Functions of Polymers with and without Interactions. I. The Distribution Function of the Square Distance of the Center of Mass from One Fixed End of a Polymer Chain
}

\author{
Takao Minato and Akira Hatano \\ Department of Pure and Applied Sciences, College of General Education, \\ University of Tokyo, Komaba, Meguro-ku, Tokyo 153, Japan.
}

(Received July 20, 1976)

\begin{abstract}
The non-Gaussian distribution function of the square distance of the center of mass from one fixed end of a polymer chain is discussed in detail. The characteristic functions of the distribution function with and without interactions are represented by the Feynman path integral.

The path integral is evaluated in closed form exactly for the non-interacting case and approximately for the interacting case by the use of a trial path integral. The distribution function and some statistical quantities are calculated analytically and numerically in two dimensions. It is shown that the most probable peak of the distribution function is pushed out and pulled in by repulsive forces and attractive forces, respectively.

KEY WORDS Distribution / Interaction / Path Integral / Center of Mass / Chain /
\end{abstract}

The excluded volume effect in polymer statistics is one of the most difficult unsolved problem. $^{1}$ The distribution function $P(\boldsymbol{R}, L)$ of the end-to-end distance $\boldsymbol{R}$ and the mean square end-to-end distance $\left\langle\boldsymbol{R}^{2}\right\rangle$ of a linear polymer chain with an excluded volume, where $L$ is the total length of the chain, has been studied theoretically and experimentally from various points of view by many investigators. ${ }^{2-7}$ Computer experiments (Monte Carlo Method ${ }^{8-10}$ and Exact Enumeration Method ${ }^{11-13}$ ) lead to the conclusion that the dependence of $\left\langle\boldsymbol{R}^{2}\right\rangle$ on $L$ may be expressed as $\left\langle\boldsymbol{R}^{2}\right\rangle \propto L^{\nu}$, where the exponent $\nu$ has the value of $1.2-1.333$ in three-dimensional space and depends only on the space dimensionality and the intra-chain potential range.

Interesting information concerning the behavior of $P(R, L)$ has been given by many studies. ${ }^{8,11}$ Several authors have given analytic representations for $P(R, L) .^{2,4,14}$ Many of these studies indicate that the well-known Gaussian nature of the distribution is destroyed completely if interaction forces are taken into account. The important influences of the excluded volume on the Gaussian nature appear in two respects; one is that the length of a segment changes from the original length to the longer renormalized one owing to many body interactions between segments, and the other is that the most probable peak of $P(R, L)$ shifts out because each segment pushes away from the others faster than in the ideal random configuration.

There are other measurable quantities besides $P(\boldsymbol{R}, L)$ and $\left\langle\boldsymbol{R}^{2}\right\rangle$; the mean square radius of gyration $\left\langle S^{2}\right\rangle$, the mean square distance of the center of mass from one fixed end, which is a special case of $\left\langle\boldsymbol{S}^{2}\right\rangle$, and their distribution functions. $\left\langle\boldsymbol{S}^{2}\right\rangle$ and the distribution function $P\left(S^{2}\right)$ of the square radius of gyration have been studied over the last several years by many workers. ${ }^{15-20}$ This is not unexpected because of the vital role that this distribution function plays in various aspects of polymer science. The problem of determining $P\left(S^{2}\right)$ for the linear chain has been approached from various points of view. Forsman and Hughes ${ }^{17}$ have formulated the problem in terms of a series of convolution integrals in order to investigate the chain of a finite length, and have given approximate solutions for various ranges of $S^{2}$. Fixman ${ }^{15}$ 
and Coriell and Jackson ${ }^{18}$ have obtained the Fourier (Laplace) transform of the distribution function $P\left(\boldsymbol{S}^{2}\right)$ and used different mathematical techniques to give approximate solutions.

Later, Fujita and Norisue ${ }^{21}$ have inverted Fixman's integral analytically in terms of Bessel functions for low and high values of $S^{2} /\left\langle S^{2}\right\rangle_{0}$ in three-dimensional space (where $\left\langle\boldsymbol{S}^{2}\right\rangle_{0}$ is the unperturbed mean square radius of gyration). Koyama has evaluated it numerically on an electronic computer in three-dimensional space. ${ }^{22}$ It seems from these studies that the behavior of $P\left(S^{2}\right)$ and that of $\left\langle S^{2}\right\rangle$ without interactions are known sufficiently. The important result is that the distribution function $P\left(S^{2}\right)$ is not, in contrast to $P(R, L)$, a Gaussian distribution over the entire range of $S^{2}$.

Therefore, it would be a very interesting problem to investigate the effect of interactions on non-Gaussian distribution functions. Such studies have not yet been presented, as far as the authors know. Though there have been some discussions restricted to perturbational approaches of the excluded volume for $\left\langle\boldsymbol{S}^{2}\right\rangle$, very little work has been reported on the perturbed distribution function $P\left(S^{2}\right){ }^{1,23}$

In this paper, we have applied the Feymnan path integral method to study the effect of intrachain interactions on $P\left(S^{2}\right)$ and the other nonGaussian distribution functions. The integration in function space (path integral) introduced by Wiener $^{24}$ and Feynman ${ }^{25}$ has become a very powerful tool in many branches of physics. ${ }^{26,27}$ To simplify the problem, in this paper we have discussed the distribution function of the square distance of the center of mass from one fixed end of a polymer chain, which has essentially the same nature as $P\left(S^{2}\right)$.

First, the characteristic function (the Fourier transform of the distribution function) is represented by the path integral. Secondly, the path integral is evaluated with some suitable boundary conditions. Thirdly, the Fourier transform of the characteristic function is performed analytically and numerically in two-dimensional space.

\section{FOUNDATION}

Path Integral Representation of the Characteristic Function

Edwards $^{2}$ and Freed $^{26}$ have expressed every possible configuration of a single polymer chain which has the total length of $L$ as a continuous space curve $\boldsymbol{r}_{t}$, where $\boldsymbol{r}_{t}$ is the $d$-dimensional position vector of a portion that is the contour length of $t$ apart from the origin $r_{0}=0$ ( $t$ varies from 0 to $L)$. They have given the Gaussian probability $P\left[\boldsymbol{r}_{t}\right]$ for the particular configuration $\boldsymbol{r}_{t}$ which lies between $\boldsymbol{r}_{t}+\delta \boldsymbol{r}_{t}$ and $\boldsymbol{r}_{t}$. This is written as

$$
P\left[\boldsymbol{r}_{t}\right] \delta \boldsymbol{r}_{t} \propto \exp \left[-\frac{d}{2 l} \int_{0}^{L} \mathrm{~d} t \dot{\boldsymbol{r}}_{t}^{2}\right] \delta \boldsymbol{r}_{t}
$$

where $l$ is the length of the segment. Hereafter $d / 2 l$ is replaced by $A$. The particular probability that the end-to-end vector is $R$ is given by the integration of eq 1 over all paths under the conditions that $\boldsymbol{r}_{0}=\mathbf{0}$ and $\boldsymbol{r}_{L}=\boldsymbol{R}$. In this case, the distribution function $P(\boldsymbol{R}, L)$ is written in the form:

$$
P(\boldsymbol{R}, L) \propto \int_{r_{0}=0}^{r_{L}=R} \mathscr{D} \boldsymbol{e x p}\left(-A \int_{0}^{L} \mathrm{~d} t \dot{\boldsymbol{r}}_{t}^{2}\right)
$$

The partition function $Z(L)$ is also given by

$$
\begin{aligned}
Z(L) & \propto \int_{\mathrm{d}^{d} \boldsymbol{R} \int_{r_{0}=0}^{r_{L}=\boldsymbol{R}} \mathscr{D} \exp \left(-A \int_{0}^{L} \mathrm{~d} t \dot{\boldsymbol{r}}_{t}^{2}\right)} \\
& \equiv \int_{r_{0}=0} \mathscr{D} \exp \left(-A \int_{0}^{L} \mathrm{~d} t \dot{\boldsymbol{r}}_{t}^{2}\right)
\end{aligned}
$$

When there are interactions between segments which have the form of $V\left(\left|\boldsymbol{r}_{t}-\boldsymbol{r}_{s}\right|\right)$, we must add an extra term of

$$
\frac{1}{2 l^{2}} \iint_{0}^{L} \mathrm{~d} t \mathrm{~d} s V\left(\left|\boldsymbol{r}_{t}-\boldsymbol{r}_{\boldsymbol{s}}\right|\right)
$$

to the exponential argument in eq 4. Thus, eq 4 becomes

$$
\begin{array}{r}
Z(L) \propto \int \mathrm{d}^{d} R \int_{r_{0}=0}^{r_{L}=\boldsymbol{R}} \mathscr{D} \boldsymbol{r} \exp \left[-A \int_{0}^{L} \mathrm{~d} t \dot{\boldsymbol{r}}_{t}^{2}\right. \\
\left.-B \iint_{0}^{L} \mathrm{~d} t \mathrm{~d} s V\left(\left|\boldsymbol{r}_{t}-\boldsymbol{r}_{s}\right|\right)\right]
\end{array}
$$

Further, if we want to calculate the distribution function $P\left(S^{2}\right)$ of the square radius of gyration $S^{2}$ or other statistical quantities, we must take into account other suitable conditions 
when we integrate eq 6 over all paths, as first shown by Fixman. As an example, the square radius of gyration is given by

$$
\boldsymbol{S}^{2}=\frac{1}{2 L^{2}} \iint_{0}^{L} \mathrm{~d} t \mathrm{~d} s\left(\boldsymbol{r}_{t}-\boldsymbol{r}_{s}\right)^{2}
$$

Taking into account the above condition, we have

$$
\begin{aligned}
P\left(\boldsymbol{S}^{2}\right)= & \int_{r_{0}=0} \mathscr{D} \boldsymbol{r} \delta\left[\boldsymbol{S}^{2}-\frac{1}{2 L^{2}} \iint_{0}^{L} \mathrm{~d} t \mathrm{~d} s\left(\boldsymbol{r}_{t}-\boldsymbol{r}_{s}\right)^{2}\right] \\
& \times \exp \left[-A \int_{0}^{L} \mathrm{~d} t \dot{\boldsymbol{r}}_{t}{ }^{2}\right. \\
& \left.-B \iint_{0}^{L} \mathrm{~d} t \mathrm{~d} s V\left(\left|\boldsymbol{r}_{t}-\boldsymbol{r}_{s}\right|\right)\right]
\end{aligned}
$$

for the distribution function $P\left(S^{2}\right)$, where $B=$ $1 / 2 l^{2}$. Introducing characteristic functions $K(\boldsymbol{R}$, $L ; \eta)$ and $K(L ; \eta)$, we may rewrite eq. 8 in the form

$$
\begin{aligned}
P\left(\boldsymbol{S}^{2}\right) & =\int_{-\infty}^{+\infty} \frac{\mathrm{d} \eta}{2 \pi} \mathrm{e}^{-i \eta S^{2}} K(L, \eta) \\
& =\int_{-\infty}^{+\infty} \frac{\mathrm{d} \eta}{2 \pi} \mathrm{e}^{-i \eta S^{2}} \int \mathrm{d}^{d} \boldsymbol{R} K(\boldsymbol{R}, L ; \eta)
\end{aligned}
$$

with

$$
\begin{aligned}
K(\boldsymbol{R}, L ; \eta)= & \int_{r_{0}=0}^{r_{L}=\boldsymbol{R}} \mathscr{D} \boldsymbol{e x p}\left[-A \int_{0}^{L} \mathrm{~d} \dot{\boldsymbol{r}}_{t}{ }^{2}\right. \\
& +\frac{i \eta}{2 L^{2}} \iint_{0}^{L} \mathrm{~d} t \mathrm{~d} s\left(\boldsymbol{r}_{t}-\boldsymbol{r}_{s}\right)^{2} \\
& \left.-B \iint_{0}^{L} \mathrm{~d} t \mathrm{~d} s V\left(\left|\boldsymbol{r}_{t}-\boldsymbol{r}_{s}\right|\right)\right]
\end{aligned}
$$

and

$$
K(L ; \eta)=\int \mathrm{d}^{d} R K(\boldsymbol{R}, L ; \eta)
$$

If the interaction term of $V\left(\left|\boldsymbol{r}_{t}-\boldsymbol{r}_{s}\right|\right)$ does not exist, $K(L ; \eta)$ can be evaluated directly without the calculation of $K(R, L ; \eta)$ as shown by Fixman ${ }^{15}$ and Coriell and Jackson. ${ }^{18}$ The characteristic function $K(\boldsymbol{R}, L ; \eta)$ can also be calculated exactly with somewhat difficult procedures, but essentially the same procedures which will be mentioned in a later section..$^{28-30,38,39}$ But we can make some simplifications without loss of generality, that is, we neglect the cross term of $\boldsymbol{r}_{t} \cdot \boldsymbol{r}_{\boldsymbol{s}}$ in eq 10 and 11. The path integral representations defined in such a way are equivalent to those for the characteristic function of the distribution function of the square distance of the center of mass from one fixed end of a polymer chain.
To distinguish these from eq 10 and 11, we denote these by adding the subscript argument $\mathrm{G}$ to give

$$
\begin{aligned}
K_{G}(\boldsymbol{R}, L ; \eta)= & \int_{r_{0}=0}^{\boldsymbol{r}_{L}=\boldsymbol{R}} \mathscr{D} \boldsymbol{r} \exp \left[-A \int_{0}^{L} \mathrm{~d} t \dot{\boldsymbol{r}}_{t}{ }^{2}\right. \\
& +\frac{i \eta}{L} \int_{0}^{L} \mathrm{~d} t \boldsymbol{r}_{t}{ }^{2} \\
& \left.-B \iint_{0}^{L} \mathrm{~d} t \mathrm{~d} s V\left(\left|\boldsymbol{r}_{t}-\boldsymbol{r}_{s}\right|\right)\right]
\end{aligned}
$$

and

$$
K_{\mathrm{G}}(L ; \eta)=\int \mathrm{d}^{d} R K_{\mathrm{G}}(\boldsymbol{R}, L ; \eta)
$$

The above expressions are those we will consider in a later section.

\section{CALCULATIONS}

\section{Calculation of the Path Integral: Ideal Case}

If the interaction term of $V\left(\left|\boldsymbol{r}_{t}-\boldsymbol{r}_{\boldsymbol{s}}\right|\right)$ does not exist, that is, in the case of the ideal chain, eq 12 and 13 are the path integral representations for the particle bound to the complex harmonic potential. This is an exactly soluble problem. We next have two formulas in $d$ dimensional space (see Appendix 1):

$$
K_{\mathrm{G}}{ }^{0}(\boldsymbol{R}, L ; \eta)=(x / \sin x)^{d / 2} \exp \left[-\left(\frac{A \boldsymbol{R}^{2}}{L}\right) x \cot x\right]
$$

and

$$
K_{\mathrm{G}}{ }^{0}(L ; \eta)=(1 / \cos x)^{d / 2}
$$

where $x$ is defined by the equation

$$
x^{2}=i \eta L / A
$$

Here, we can consider the following three cases: 1) the linear chain with the end-to-end vector $R$ fixed, 2) the ring chain given by $R=0$ in eq 14 , and 3 ) the linear random flight chain which is given from eq 15 . For all cases, the moments $\left\langle\boldsymbol{S}^{2}\right\rangle$ can be calculated by the method of cumulants. The calculations are very easy, so we will not reproduce them here.

Now, we will evaluate the distribution function $P_{G}{ }^{0}\left(S^{2}\right)$ by performing the Fourier transform of the characteristic function analytically. Case 1) is too complicated for us to be able to carry out the Fourier transform analytically. Case 2) reduces to the famous Fixman's result ${ }^{15}$ and has 


\section{T. Minato and A. Hatano}

been discussed by many investigators. Therefore, we consider case 3 ) by the use of a saddle point method and a contour integral.

From eq 9 and 15, we have

$$
\begin{aligned}
P_{\mathrm{G}}{ }^{0}\left(S^{2}\right) & =\int_{-\infty}^{+\infty} \frac{\mathrm{d} \eta}{2 \pi} \mathrm{e}^{-i \eta S^{2}} K_{G}{ }^{0}(L ; \eta) \\
& \equiv \int_{-\infty}^{+\infty} \frac{\mathrm{d} \eta}{2 \pi}\left(\frac{1}{\cos x}\right)^{d / 2} \exp \left(-d \xi x^{2} / 4\right) \\
& \equiv \int_{-\infty}^{+\infty} \frac{\mathrm{d} \eta}{2 \pi} \exp [-g(\eta)]
\end{aligned}
$$

with

$$
g(\eta)=d \xi x^{2} / 4+(d / 2) \ln \cos x
$$

where $\xi=4 A S^{2} / d L=2 S^{2} / l L$.

If the saddle point $x^{*}\left(\eta^{*}\right)$ is determined for various ranges of $\xi, P_{G}{ }^{0}\left(S^{2}\right)$ is given by the following equation:

$$
\begin{aligned}
P_{\mathrm{G}}{ }^{0}\left(\boldsymbol{S}^{2}\right) & =\int_{-\infty}^{+\infty} \frac{\mathrm{d} \eta}{2 \pi} \exp \left[-g\left(\eta^{*}\right)-g^{\prime \prime}\left(\eta^{*}\right)\left(\eta-\eta^{*}\right)^{2} / 2\right] \\
& \equiv\left[2 \pi g^{\prime \prime}\left(\eta^{*}\right)\right]^{-1 / 2} \exp \left[-g\left(\eta^{*}\right)\right]
\end{aligned}
$$

We can consider three cases: they are $\xi \simeq 1, \xi 》$ 1 , and $\xi \ll 1$ for $d$-dimensional space.

1. Near the Mean $\xi=1$. The saddle point $x^{*}\left(\eta^{*}\right)$ is the root of the following equation:

$$
\xi x-\tan x=0
$$

Put $\xi=1+\varepsilon$, and suppose $\varepsilon \ll 1$. Then $x^{*}$ will be small. Eq 20 gives

$$
\eta^{*}=-3 i \varepsilon A / L ; \quad x^{* 2}=3 \varepsilon
$$

Thus for $\xi>1$, the saddle point is on the negative imaginary axis of the complex $\eta$ plane, and on the positive imaginary axis for $\xi<1$. By taking the contour passing through $\eta^{*}$ parallel to the real axis, eq 19 becomes

$$
\left\langle S^{2}\right\rangle P_{\mathrm{G}}{ }^{0}\left(\boldsymbol{S}^{2}\right)=(d / 4)(6 / \pi d)^{1 / 2} \exp \left[-3 d(1-\xi)^{2} / 8\right]
$$

where $\left\langle\boldsymbol{S}^{2}\right\rangle=l L / 2$.

2. Large $S^{2} ; \xi \gg 1$. If $\xi$ is large, the root of eq 20 will occur near $x=\pi / 2$. In eq 20 , put $x=\pi / 2-\delta$ and suppose $\delta$ to be small; then eq 20 gives a saddle point at

$$
\delta=2 /(\pi \xi)
$$

Since the contour can be deformed to pass through the saddle point without crossing any singular point or branch line, eq 19 gives

$$
\begin{aligned}
\left\langle S^{2}\right\rangle P_{\mathrm{G}}{ }^{0}\left(S^{2}\right)= & (d / 2)(1 / \pi d)^{1 / 2}(2 / \pi \xi)^{-d / 2} \xi^{-1} \\
& \times \exp \left[-d \pi^{2} \xi / 16\right]
\end{aligned}
$$

3. Small $S^{2} ; 0<\xi \ll 1$. For $\xi \ll 1$, the saddle point is far out on the positive imaginary axis of the complex $\eta$ plane. Equation 20 yields as the saddle point

$$
\eta^{*}=i A / L \xi^{2} ; \quad x^{*}=i / \xi
$$

Therefore, the result of the steepest descent integration is, from eq 19 and 25 ,

$$
\left\langle\boldsymbol{S}^{2}\right\rangle P_{\mathrm{G}}{ }^{0}\left(\boldsymbol{S}^{2}\right)=2^{d / 2}(\pi d)^{-1 / 2}(d / 2) \xi^{-3 / 2} \exp (-d / 4 \xi)
$$

\section{for small $\xi$.}

We will next derive rigorous expressions for $\left\langle S^{2}\right\rangle P_{G}{ }^{0}\left(S^{2}\right)$ valid for large $\xi$ and small $\xi$ in two-dimensional space by the use of the contour integration used by Fujita and Norisue ${ }^{21}$ and Sanchez and Frankenberg. ${ }^{32}$ We may rewrite eq 17 in the following form in two-dimensional space $(d=2)$ :

$$
\left\langle S^{2}\right\rangle P_{G}{ }^{0}\left(S^{2}\right)=\frac{1}{2 \pi i} \int_{\Gamma} \mathrm{d} x(x / \cos x) \exp \left(-\xi x^{2} / 2\right)
$$

where $\Gamma$ is the path of integration as shown in Fig. 4 in Appendix 2. The asymptotic form of $\left\langle S^{2}\right\rangle P_{G}{ }^{0}\left(S^{2}\right)$ valid for small $S^{2}$ is given by the method of Fujita and Norisue. In their treatment, the original path $\Gamma$ is deformed to $U\left(S_{1} K_{1} D_{1} D_{2} K_{2} S_{2}\right)$ as is illustrated in Figure 4. This leads immediately to the following asymptotic form for $d=2$;

$$
\begin{aligned}
\left\langle\boldsymbol{S}^{2}\right\rangle P_{\mathrm{G}}{ }^{0}\left(\boldsymbol{S}^{2}\right)= & \sum_{n=0}(-1)^{n}(2 n+1)(2 / \pi)^{1 / 2} \xi^{-3 / 2} \\
& \times \exp \left[-(2 n+1)^{2} / 2 \xi\right]
\end{aligned}
$$

The asymptotic form of $\left\langle S^{2}\right\rangle P_{\mathrm{G}}{ }^{0}\left(S^{2}\right)$ valid for large $S^{2}$ is given by the residue theorem. ${ }^{32}$ In this case, we add the quarter circle of radius $R^{\prime}$ to the path $\Gamma$ as shown in Figure 4. In the region enclosed by $\Gamma_{1} \Gamma_{2} S$, the integrand in eq $17^{\prime}$ has poles at $x=\left(n+\frac{1}{2}\right) \pi(n=0,1,2, \cdots)$ along the positive real axis. By the residue theorem, this leads to

$$
\begin{aligned}
\left\langle S^{2}\right\rangle P_{\mathrm{G}}{ }^{0}\left(\boldsymbol{S}^{2}\right)= & \sum_{n=0}\left(n+\frac{1}{2}\right) \pi(-1)^{n} \\
& \times \exp \left[-\left(n+\frac{1}{2}\right)^{2} \pi^{2} \xi / 2\right]
\end{aligned}
$$

The leading term of eq 27 agrees with eq 26 , 
which was derived by the method of steepest descent. But the leading term of eq 28 agrees with eq 24 only approximately.

Calculation of the Path Integral: Non Ideal Case

When the interaction term of $V\left(\left|\boldsymbol{r}_{t}-\boldsymbol{r}_{\boldsymbol{s}}\right|\right)$ exists in eq 12 and 13, these path integrals cannot be evaluated exactly; however, several mathematical devices have been employed to calculate them. ${ }^{2,26,27}$ We will apply one of them, which was originally proposed by Feynman in his theory of polarons. ${ }^{31}$ We define a true action $S$ by the equation,

$$
\begin{aligned}
K_{\mathrm{G}}(\boldsymbol{R}, L ; \eta)= & \int_{r_{0}=0}^{r_{L}=\boldsymbol{R}} \mathscr{D} \exp (-S) \\
\equiv & \int_{r_{0}=0}^{r_{L}=\boldsymbol{R}} \mathscr{D} \boldsymbol{r e x p}\left[-A \int_{0}^{L} \mathrm{~d} t \dot{\boldsymbol{r}}_{t}{ }^{2}\right. \\
& +\frac{i \eta}{L} \int_{0}^{L} \mathrm{~d} t \boldsymbol{r}_{t}{ }^{2} \\
& \left.-B \iint_{0}^{L} \mathrm{~d} t \mathrm{~d} s V\left(\left|\boldsymbol{r}_{t}-\boldsymbol{r}_{\boldsymbol{s}}\right|\right)\right]
\end{aligned}
$$

Introducing a trial action $S_{0}$, we rewrite eq 29 formally in the form

$$
\begin{aligned}
K_{G}(\boldsymbol{R}, L ; \eta) & =\int \mathscr{D} \boldsymbol{r} \mathrm{e}^{-\left(S-S_{0}\right)} \mathrm{e}^{-S_{0}} \\
& =\left\langle\mathrm{e}^{-\left(S-S_{0}\right)}\right\rangle \int \mathscr{D} \boldsymbol{r} \mathrm{e}^{-S_{0}}
\end{aligned}
$$

where $\langle\cdots\rangle$ is an average by the path integral with $S_{0}$, i.e.,

$$
\langle\cdots\rangle=\int \mathscr{D} \boldsymbol{r} \cdots \mathrm{e}^{-S_{0}} / \int \mathscr{D} \boldsymbol{r} \mathrm{e}^{-S_{0}}
$$

Here, we make the approximation that

$$
\left\langle\exp \left[-\left(S-S_{0}\right)\right]\right\rangle \simeq \exp \left[-\left\langle\left(S-S_{0}\right)\right\rangle\right]
$$

Then, eq 29 can be calculated approximately, if an exactly calculable action $S_{0}$ is being used. As a trial action $S_{0}$, we choose

$$
S_{0}=A \int_{0}^{L} \mathrm{dt} \dot{r}_{t}{ }^{2}-\frac{i \eta}{L} \int_{0}^{L} \mathrm{~d} t r_{t}^{2}
$$

Then, the factor $\left\langle S-S_{0}\right\rangle$ in eq 32 is given by

$$
\begin{aligned}
\left\langle S-S_{0}\right\rangle & =B\left\langle\iint_{0}^{L} \mathrm{~d} t \mathrm{~d} s V\left(\left|\boldsymbol{r}_{t}-\boldsymbol{r}_{s}\right|\right)\right\rangle \\
& \equiv B \iiint_{0}^{L} \mathrm{~d} t \mathrm{~d} s \mathrm{~d}^{d} k V(\boldsymbol{k})\left\langle\exp i \boldsymbol{k}\left(\boldsymbol{r}_{t}-\boldsymbol{r}_{s}\right)\right\rangle
\end{aligned}
$$

where an average is defined by eq 31 , and $V(\boldsymbol{k})$ is given by the equation,

$$
V(\boldsymbol{k})=\frac{1}{(2 \pi)^{d}} \int \mathrm{d}\left(\left|\boldsymbol{r}_{t}-\boldsymbol{r}_{s}\right|\right) V\left(\left|\boldsymbol{r}_{t}-\boldsymbol{r}_{s}\right|\right) \exp i \boldsymbol{k}\left(\boldsymbol{r}_{t}-\boldsymbol{r}_{s}\right)
$$

Here, we rewrite the exponential factor in eq 34 in the form:

$$
\begin{aligned}
\left\langle\exp -i \boldsymbol{k}\left(\boldsymbol{r}_{o}-\boldsymbol{r}_{\tau}\right)\right\rangle \\
=\left[\int \mathscr { D } \boldsymbol { r } \operatorname { e x p } \left(-A \int_{0}^{L} \mathrm{~d} t \dot{\boldsymbol{r}}_{t}{ }^{2}+\frac{i \eta}{L} \int_{0}^{L} \mathrm{~d} t \boldsymbol{r}_{t}{ }^{2}\right.\right. \\
\left.\left.+i \boldsymbol{k}\left(\boldsymbol{r}_{o}-\boldsymbol{r}_{\tau}\right)\right)\right] / \int \mathscr{D} \boldsymbol{r} \exp -S_{0}
\end{aligned}
$$

The numerator of the r.h.s. of eq 36 can be written in the form:

$$
\begin{aligned}
I= & \int \mathscr{D} \boldsymbol{r} \exp \left[-A \int_{0}^{L} \mathrm{~d} t \dot{\boldsymbol{r}}_{t}{ }^{2}+\frac{i \eta}{L} \int_{0}^{L} \mathrm{~d} t \boldsymbol{r}_{t}{ }^{2}\right. \\
& \left.+\int_{0}^{L} \mathrm{~d} t \boldsymbol{f}_{t} \cdot \boldsymbol{r}_{t}\right]
\end{aligned}
$$

with

$$
\boldsymbol{f}_{t}=i \cdot \boldsymbol{k} \cdot[\delta(t-\sigma)-\delta(t-\tau)]
$$

We have applied the method of Laplace to calculate the path integral $I$. Since the contribution of path $\boldsymbol{r}_{t}$ to $I$ becomes most dominant when $\boldsymbol{r}_{t}$ makes the exponential argument in eq 37 stationary, we get $\boldsymbol{r}_{t}$ as a solution of the following Euler-Lagrange equation:

with

$$
\ddot{\boldsymbol{r}}_{t}-\alpha^{2} \boldsymbol{r}_{t}+\beta \boldsymbol{f}_{t}=0
$$

$$
\alpha^{2}=-i \eta / A L, \quad \text { and } \quad \beta=1 / 2 A
$$

By taking this solution, $I$ is given by

$$
I=\left[-\dot{\boldsymbol{r}}_{0} \boldsymbol{r}_{0}+\dot{\boldsymbol{r}}_{L} \boldsymbol{r}_{L}+\frac{1}{2} \int_{0}^{L} \mathrm{~d} t \boldsymbol{f}_{t} \cdot \boldsymbol{r}_{t}\right]
$$

Now, the solution of eq 39 has the form

$$
\boldsymbol{r}_{t}=W \mathrm{e}^{\alpha t}+Q \mathrm{e}^{-\alpha t}+(\beta / 2 \alpha) \int_{0}^{L} \mathrm{~d} s f_{s} \cdot \mathrm{e}^{-\alpha|t-s|}
$$

where $W$ and $Q$ are constants determined from the boundary condition on $\boldsymbol{r}_{t}$ subjected to $\boldsymbol{r}_{0}=$ $\boldsymbol{r}_{L}=0$. This boundary condition corresponds to the case of the ring chain. From the cyclic boundary condition and the condition of $\boldsymbol{r}_{t}$ being finite in the limit of $L \rightarrow \infty$, we obtain the result: $\boldsymbol{r}_{t}=(i \boldsymbol{k} \beta / 2 \alpha)\left[\left(\mathrm{e}^{-\alpha \sigma}-\mathrm{e}^{-\alpha \tau}\right) \mathrm{e}^{-\alpha t}-\left(\mathrm{e}^{-\alpha|t-\sigma|}-\mathrm{e}^{-\alpha|t-\tau|}\right)\right]$ 
under the condition of Re. $\alpha>0$ (Real part of $\alpha$ is positive). Substituting eq 43 into eq 41 , we have

$$
I=\exp \left\{-(\beta / 4 \alpha)\left[2\left(1-\mathrm{e}^{-\alpha|\sigma-\tau|}\right)-\left(\mathrm{e}^{-\alpha \sigma}-\mathrm{e}^{-\alpha \tau}\right)^{2}\right] k^{2}\right\}
$$

Using eq 34-36, 44 and the pseduo-potential for $V(\boldsymbol{r})$, that is, $V(\boldsymbol{r})=V \delta(\boldsymbol{r})$, we have

$$
\begin{aligned}
\left\langle S-S_{0}\right\rangle= & \frac{B V}{(2 \pi)^{d}} \iiint_{0}^{L} \mathrm{~d} \sigma \mathrm{d} \tau \mathrm{d}^{d} k \\
& \times \exp \left\{-(\beta / 4 \alpha)\left[2\left(1-\mathrm{e}^{-\alpha|\sigma-\tau|}\right)\right.\right. \\
& \left.\left.-\left(\mathrm{e}^{-\alpha \sigma}-\mathrm{e}^{-\alpha \tau}\right)^{2}\right] k^{2}\right\}
\end{aligned}
$$

Performing the integration over $\boldsymbol{k}$ in $d$-dimensional space, we have

$$
\begin{aligned}
\left\langle S-S_{0}\right\rangle= & \frac{B V}{2^{d} \pi^{d / 2}}(4 \alpha / \beta)^{d / 2} \iint_{0}^{L} \mathrm{~d} \sigma \mathrm{d} \tau\left[2\left(1-\mathrm{e}^{-\alpha|\sigma-\tau|}\right)\right. \\
& \left.\times\left(\mathrm{e}^{-\alpha \sigma}-\mathrm{e}^{-\alpha \tau}\right)^{2}\right]^{-d / 2}
\end{aligned}
$$

In the limit of $L \rightarrow \infty$, eq 46 can be approximated as

$$
\begin{aligned}
\langle S & \left.-S_{0}\right\rangle \\
& =\frac{B V L^{2}}{2^{d-1} \pi^{d / 2}}(4 \alpha / \beta)^{d / 2} \int_{0}^{1} \mathrm{~d} y(1-y)\left[2\left(1-\mathrm{e}^{-\alpha L y}\right)\right]^{-d / 2} \\
& \simeq \frac{B V L^{2}}{(2 \pi)^{d / 2}}(\alpha / \beta)^{d / 2}
\end{aligned}
$$

where $\alpha$ must satisfy the condition of Re. $\alpha>0$.

Now, from eq 16 and 40 , the following relation can be derived:

$$
\alpha^{2}=-x^{2} / L^{2}
$$

Taking the condition of Re. $\alpha>0$ into account, we have the following two solutions:

and

$$
\left.\begin{array}{ll}
\alpha=-i x / L & \text { for Im. } x>0 \\
\alpha=+i x / L & \text { for } \operatorname{Im} . x<0
\end{array}\right\}
$$

From these results eq 47 can be written in terms of $x$ in the form:

$$
\left\langle S-S_{0}\right\rangle=v( \pm i x)^{d / 2}
$$

where $v=B V L^{(4-d) / 2} /(2 \pi \beta)^{d / 2} ;(-)$ corresponds to the case of Im. $x>0$ and (+) to that of Im. $x<0$.

As a final expression for the characteristic function $K_{\mathrm{G}}(0, L ; \eta)$ of the ring chain, from eq 14,28 , and 50 , we have

$$
K_{\mathrm{G}}(0, L ; \eta)=(x / \sin x)^{d / 2} \exp \left[-v( \pm i x)^{d / 2}\right]
$$

As seen from eq 51, we cannot calculate the Fourier transform of $K_{G}(0, L ; \eta)$ analytically except for $d=2$ and $d=4$. In four-dimensional space, we can carry out the same procedure as mentioned before. Apparently $P_{G}\left(S^{2}\right)$ does in effect behave like the ideal distribution function $P_{G}{ }^{0}\left(S^{2}\right)$. The second moments $\left\langle S^{2}\right\rangle$ can be given by the method of cumulants as follows:

$$
\left\langle\boldsymbol{S}^{2}\right\rangle=\left(\frac{1}{3}+v\right) L / A
$$

The dependence of $\left\langle S^{2}\right\rangle$ on $L$ is the same as for a Gaussian chain. The effect of interactions disappears in four-dimensional space in this approximation. In addition, since $K_{G}(0, L ; \eta)$ is nonanalytic with respect to $\eta$ except at $d=4$, the mements $\left\langle\boldsymbol{S}^{2 n}\right\rangle$ ( $n$ is an integer) cannat be obtained by the method of cumulants. It is required to perform the Fourier transform explicitly in order to obtain information about the moments of the distribution.

\section{RESULTS}

\section{Fourier Transform of the Characteristic Function: Analytical}

From eq 9 and 51, the distribution function $P_{G}\left(S^{2}\right)$ can be written in the form:

$$
\begin{aligned}
\left\langle S^{2}\right\rangle P_{\mathrm{G}}\left(\boldsymbol{S}^{2}\right)= & \frac{d}{12 i \pi} \int_{\Gamma} \mathrm{d} x x(x / \sin x)^{d / 2} \\
& \times \exp \left[-d \xi x^{2} / 12-v( \pm i x)^{d / 2}\right]
\end{aligned}
$$

where $\xi=6 S^{2} / l L,\left\langle S^{2}\right\rangle=l L / 6$, and $\Gamma$ represents the path of integration (see Appendix 2). It will be impossible to calculate the above integral analytically except by using a numerical calculation. But fortunately it can be evaluated exactly in two-dimensional space $(d=2)$ by the method of the contour integrations employed by Fujita and Norisue ${ }^{21}$ in three-dimensional space. Though these calculations are very tedious, we have obtained the following two asymptotic forms (see Appendix 2):

$$
\begin{aligned}
\left\langle\boldsymbol{S}^{2}\right\rangle \boldsymbol{P}_{\mathrm{G}}\left(\boldsymbol{S}^{2}\right)= & (6 / \pi)^{1 / 2}\left[3(1+v)^{2} \xi^{-5 / 2}-\xi^{-3 / 2}\right] \\
& \times \exp \left[-3(1+v)^{2} / 2 \xi\right]
\end{aligned}
$$

for small values of $\xi$, and

$$
\begin{aligned}
\left\langle S^{2}\right\rangle P_{G}\left(S^{2}\right)= & (1 / 3) \pi^{2} \mathrm{e}^{-\pi 2 \xi / 6} \cos (\pi v) \\
& +(v / 4)(6 / \pi)^{1 / 2}\left(\xi^{-3 / 2}+(3 / 2) \xi^{-5 / 2}\right. \\
& \left.+(21 / 8) \xi^{-7 / 2}\right) \exp \left(-3 v^{2} / 2 \xi\right)
\end{aligned}
$$


for large values of $\xi$, respectively. These two expressions can be found to reduce correctly to those for the unperturbed distribution functions in two-dimenstional space if $v \rightarrow 0$ in eq $53 .^{32,38}$ We can see clearly the effect of the interactions on the unperturbed distribution function.

Fourier Transform of the Characteristic Function: Numerical Ideal Case.

Equation $17^{\prime}$ has been calculated numerically for various values of $\xi$ on an electronic computer. The results are given in Figure 1 and Table I. In Figure, 1, the distribution function $\left\langle S^{2}\right\rangle P_{G}{ }^{0}\left(S^{2}\right)$, which represents that of the square distance of the center of mass from one fixed end of the linear chain, is plotted against $\xi$; in order to compare the shape of the distribution of the linear chain and that of the ring chain, the distribution function of the ring chain which is given by putting $v=0$ in eq 53 is also plotted on the same figure. As seen from this the distribution function of the linear chain goes to zero more slowly than that of the ring chain at large values of $\xi$, but the peak of the distribution of the ring chain is farther out than that of the linear chain. The latter result is contrary to our expectations. ${ }^{39}$
Table I. The numerical results of the integration of eq $17^{\prime}$ and the comparison with analytical results from the leading term of eq 27 and eq 28 in two dimensions

\begin{tabular}{clcc}
\hline \multicolumn{4}{c}{$\left\langle\boldsymbol{S}^{2}\right\rangle \boldsymbol{P}_{\mathrm{G}}{ }^{0}\left(\boldsymbol{S}^{2}\right)$} \\
\hline$\xi$ & Num. & eq 27 & eq 28 \\
\hline 0.1 & 0.1697 & 0.1700 & 1.3884 \\
0.2 & 0.7321 & 0.7322 & 1.2273 \\
0.3 & 0.9169 & 0.9170 & 1.0848 \\
0.4 & 0.9031 & 0.9034 & 0.9589 \\
0.5 & 0.8291 & 0.8300 & 0.8476 \\
0.6 & 0.7431 & 0.7458 & 0.7492 \\
0.7 & 0.6602 & 0.6667 & 0.6623 \\
0.8 & 0.5847 & 0.5966 & 0.5854 \\
0.9 & 0.5172 & 0.5360 & 0.5174 \\
1.0 & 0.4573 & 0.4839 & 0.4574 \\
1.2 & 0.3573 & 0.3997 & 0.3574 \\
1.4 & 0.2792 & 0.3366 & 0.2792 \\
1.6 & 0.2181 & 0.2833 & 0.2182 \\
1.8 & 0.1704 & 0.2502 & 0.1704 \\
2.0 & 0.1331 & 0.2193 & 0.1332 \\
2.4 & 0.0812 & 0.1736 & 0.0813 \\
2.8 & 0.0496 & 0.1421 & 0.0496 \\
3.4 & 0.0236 & 0.1095 & 0.0236 \\
4.0 & 0.0112 & 0.0880 & 0.0112 \\
\hline
\end{tabular}

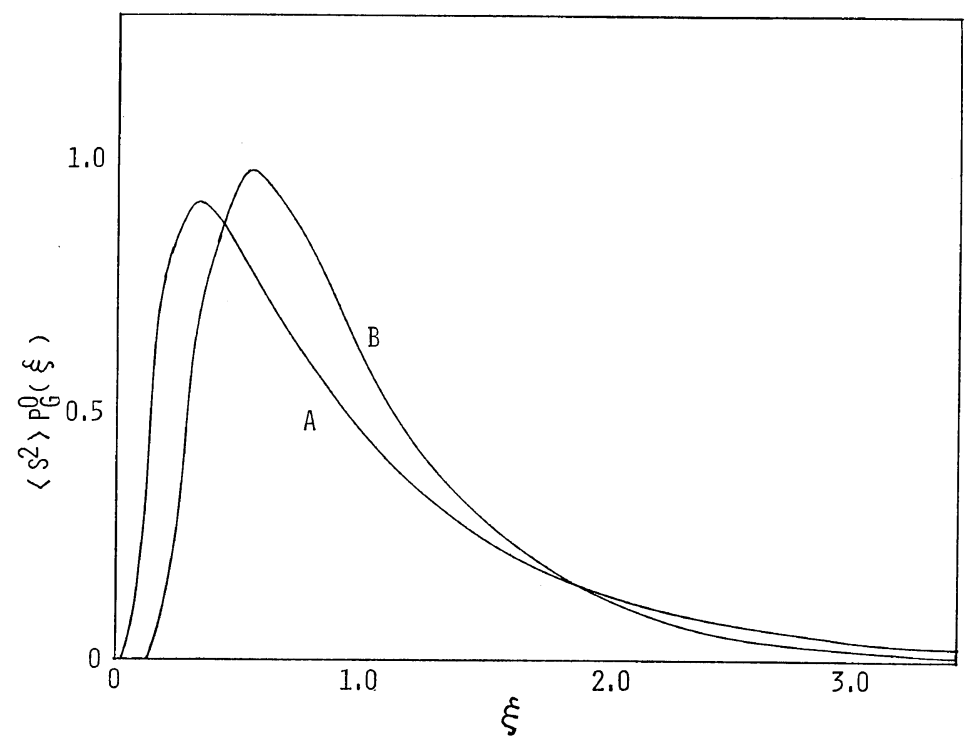

Figure 1. The two dimensional unperturbed distribution functions $\left\langle S^{2}\right\rangle P_{G}{ }^{0}(\xi)$ of a linear chain and a ring chain: curve A, linear $\left(\xi=2 S^{2} / l L\right)$; curve $B$, ring $\left(\xi=6 S^{2} / l L\right)$. 


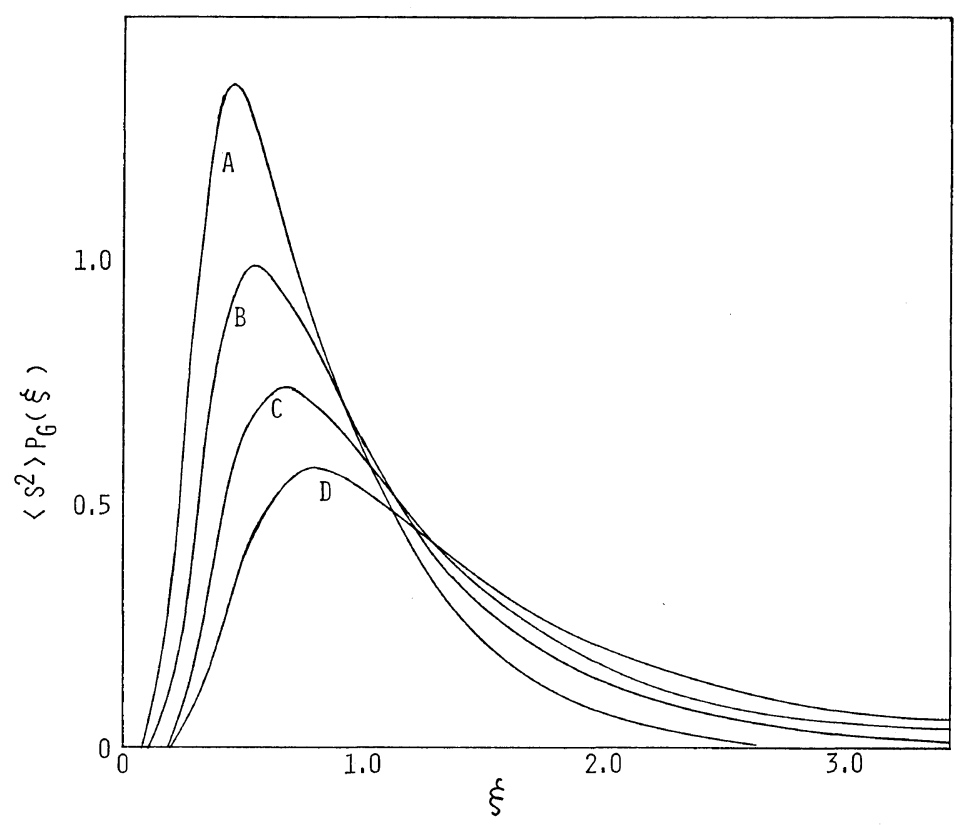

Figure 2. The two dimensional perturbed distribution functions $\left\langle\boldsymbol{S}^{2}\right\rangle \boldsymbol{P}_{\mathrm{G}}(\xi)$ of a ring chain for various values of $v$ : Curve $\mathrm{A}, v=-0.1$; curve $\mathrm{B}, v=0.0$; curve $\mathrm{C}, v=0.1$; curve $\mathrm{D}, v=0.2$.

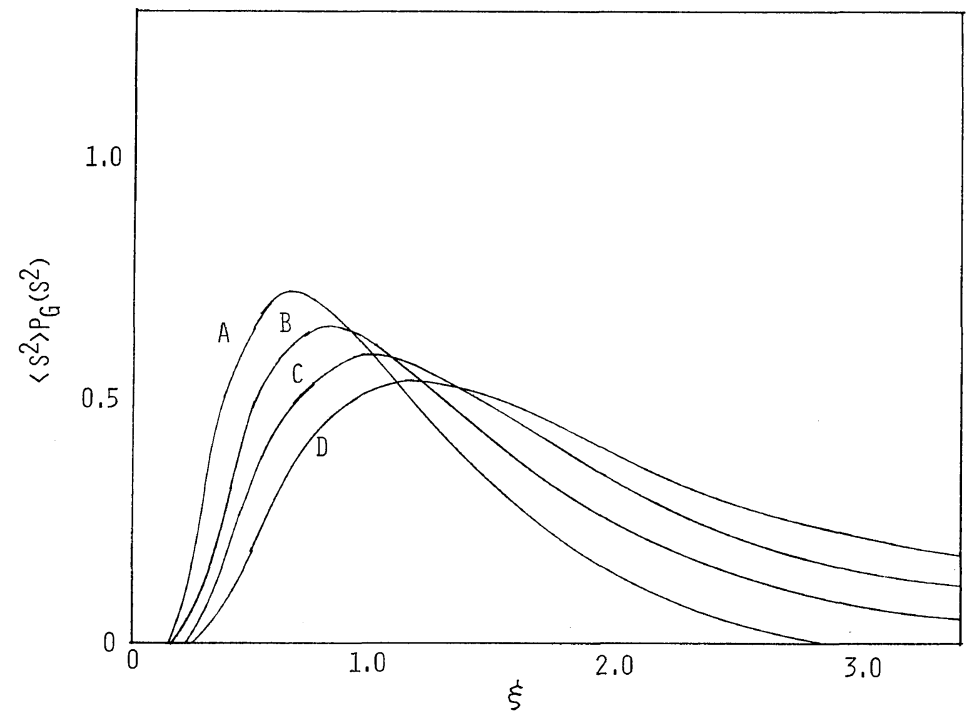

Figure 3. The two-dimensional perturbed distribution functions $\boldsymbol{S}^{2} \boldsymbol{P}_{\mathrm{G}}\left(\boldsymbol{S}^{2}\right)\left(=\left\langle\boldsymbol{S}^{2}\right\rangle \xi \boldsymbol{P}_{\mathrm{G}}(\xi)\right)$ of a ring chain: Curve $\mathrm{A}, v=-0.1$; curve $\mathrm{B}, v=0.0$; curve $\mathrm{C}, v=0.1$; curve $\mathrm{D}, v=0.2$.

Non-Ideal Case.

Equation 53 has been calculated numerically for some values of $v$ as a function of $\xi$ in two di- mensions $(d=2)$. A positive value of $v$ corresponds to the case of repulsive interactions and a negative value of $v$ to that of attractive interac- 
Table II. Results of numerical integrations of eq 53 in two dimensions

\begin{tabular}{cllll}
\hline \multicolumn{5}{c}{$\left\langle\boldsymbol{S}^{2}\right\rangle \boldsymbol{P}_{\mathrm{G}}\left(\boldsymbol{S}^{2}\right)$} \\
\hline $\boldsymbol{\xi}$ & $v=0$ & $v=-0.1$ & $v=0.1$ & $v=0.2$ \\
\hline $\mathbf{0 . 1}$ & 0.0 & 0.0017 & 0.0 & 0.0 \\
$\mathbf{0 . 2}$ & 0.1209 & 0.3915 & 0.0312 & 0.0066 \\
0.3 & 0.5100 & 1.0450 & 0.2194 & 0.0839 \\
$\mathbf{0 . 4}$ & 0.8342 & 1.3256 & 0.4721 & 0.2419 \\
0.5 & 0.9737 & 1.3309 & 0.6486 & 0.3969 \\
0.6 & 0.9754 & 1.1955 & 0.7290 & 0.5037 \\
0.7 & 0.9101 & 1.0292 & 0.7388 & 0.5574 \\
0.8 & 0.8137 & 0.8607 & 0.7065 & 0.5710 \\
0.9 & 0.7137 & 0.7137 & 0.6535 & 0.5578 \\
1.0 & 0.6162 & 0.4800 & 0.5916 & 0.5290 \\
1.2 & 0.4517 & 0.3922 & 0.4690 & 0.4517 \\
1.4 & 0.3273 & 0.2599 & 0.3637 & 0.3721 \\
1.6 & 0.2363 & 0.1702 & 0.2798 & 0.3016 \\
1.8 & 0.1703 & 0.1093 & 0.2150 & 0.2430 \\
2.0 & 0.1227 & 0.0681 & 0.1655 & 0.1956 \\
2.4 & 0.0636 & 0.0214 & 0.0995 & 0.1281 \\
2.8 & 0.0328 & 0.0007 & 0.0617 & 0.0862 \\
3.4 & 0.0121 & 0.0 & 0.0325 & 0.0509 \\
4.0 & 0.0044 & 0.0 & 0.0191 & 0.0329 \\
\hline
\end{tabular}

tions, respectively. The results are given in Figure 2 and Table II. In addition, $S^{2} P_{G}\left(S^{2}\right)$ is plotted as a function of $\xi$ in Figure 3. As seen from Figure 2 and Figure 3, the most probable peak of the distribution is pushed out and pulled in by repulsive interactions and attractive interactions, respectively. It can be shown that this numerical result is in close agreement with the analytical results from eq 54 and eq 55 in the regions where eq 54 and eq 55 are applicable.

\section{SUMMARY AND DISCUSSIONS}

We have obtained the closed characteristic function of the ring chain with intra-chain interactions on the basis of the first-order perturbation theory. The distribution function derived from the Fourier inverse transformation of the characteristic function can not be expressed in a simple function which covers the whole range of $S^{2}$. But in two dimensions analytical forms which are valid for large values of $\boldsymbol{S}^{2}$ and small values of $S^{2}$ are given by eq 54 and eq 55 . The complete solutions for arbitrary $d$ (space dimensionality) will be given by numerical integrations of eq 53 .
Here, we will calculate the second moments $\left\langle S^{2}\right\rangle$ of the distribution using eq 54 , which is applicable for small $S^{2}$. Then the second moment $\left\langle S^{2}\right\rangle$ is given by the equation:

$$
\langle\xi\rangle=\left\langle 6 S^{2} / l L\right\rangle=\int_{\propto(1+v)^{2}} \mathrm{~d} \xi \xi P_{\mathrm{G}}(\xi) / \int \mathrm{d} \xi P_{\mathrm{G}}(\xi)
$$

where $v=B V L /(2 \pi \beta)$ and $P_{G}(\xi)$ is given by eq 54. Therefore, the second moment $\left\langle S^{2}\right\rangle$ is proportional to $L\left(1+2 v+v^{2}\right)$ in two dimension. This fact shows that even though the distribution function has a closed form, the second moment does not have a closed form. This result is based on the approximation of eq 32 . In order to obtain a closed expression for the moments of the distribution, it is necessary that we use a trial path integral which contains a variational parameter and determine the variational parameter to give the minimum free energy of the chain. ${ }^{33,34}$

Finally, we note that our results obtained in this paper can be interpreted in terms of the linear chain. For the characteristic function of the square radius of gyration for the linear chain, $K_{\mathrm{L}}{ }^{0}\left(\boldsymbol{S}^{2}\right)$, has been derived by Fixman as $K_{\mathrm{L}}{ }^{0}\left(\boldsymbol{S}^{2}\right)=$ $(x / \sin x)^{d / 2}$; our result agrees with his result if we put $R=0$ in eq. 14 . On the other hand, for the interacting case, nobody has ever derived $P_{\mathrm{L}}\left(S^{2}\right)$, which is the perturbed distribution function of $S^{2}$ for the linear chain. But the behavior of $P_{\mathrm{L}}\left(S^{2}\right)$ with interactions can be obtained from eq 51, except for the numerical factor of the coefficient of $v$ in eq 51. This is because the interaction dependence of eq 50 is free from any boundary conditions.

In the future, we will consider eq 10 explicitly and attempt to use a trial path integral which contains a variational parameter. ${ }^{30}$

Acknowlegment. We would like to thank Professor R. Abe for valuable suggestions and discussion. One of the author (TM) appreciates the fruitful discussions with Mr. Y. Okabe. Numerical calculations have been made on the HITAC $8800 / 8700$ at Tokyo University.

\section{APPENDIX 1}

The method of the calculation of the path integral for the harmonic oscillator problem is 
presented in standard papers $\mathrm{s}^{26,35,36}$ and textbooks. ${ }^{37}$ Here, we derive the result by comparing eq 12 and 13 with well-known formulas of the onedimensional harmonic oscillator:

$\begin{array}{rl}\int_{y_{0}=0}^{y_{L}=R} & \mathscr{D} y \exp \left[\frac{i}{h}\left(\frac{m}{2} \int_{0}^{L} \mathrm{~d} t \dot{y}_{t}{ }^{2}-\frac{m \omega^{2}}{2} \int_{0}^{L} \mathrm{~d} t y_{t}{ }^{2}\right)\right] \\ & =\left[\frac{m \omega}{2 \pi i h \sin (\omega L)}\right]^{1 / 2} \exp \left[\frac{i m \omega R^{2}}{2 h} \cos (\omega L)\right]\end{array}$

$$
\begin{array}{r}
\int_{y_{0}=0} \mathscr{D} y \exp \left[\frac{i}{h}\left(\frac{m}{2} \int_{0}^{L} \mathrm{~d} t \dot{y}_{t}{ }^{2}-\frac{m \omega^{2}}{2} \int_{0}^{L} \mathrm{~d} t y_{t}{ }^{2}\right)\right] \\
=[\cos (\omega L)]^{-1 / 2}
\end{array}
$$

where $m, \omega, L$, and $h$ are the mass of a particle, the frequency of oscillation, time, and Planck constant, respectively. Equation A2 is derived by the integration of eqA 1 over $R$. By comparing eq 12 with eq $A 1$, we obtain the following two equations:

$$
-i m / 2 h=A ; \quad-i m \omega^{2} / 2 h=i \eta / L
$$

Therefore, we have 1.h.s. of eq 12

$$
\begin{aligned}
= & (A / \pi L)^{1 / 2}[\sqrt{(i \eta L / A)} / \sin \sqrt{(i n L / A)}]^{1 / 2} \\
& \times \exp \left[-\left(A R^{2} / L\right)(\sqrt{i \eta L / A}) \cot \sqrt{i \eta L / A}\right]
\end{aligned}
$$

$$
\text { 1.h.s. of eq } 13=[\cos \sqrt{(i \eta L / A)}]^{-1 / 2}
$$

An extra factor, $(A / \pi L)^{1 / 2}$, eq $\mathrm{A} 4$ can be dropped by imposing a normalization condition of the form:

$$
\int P_{\mathrm{G}}{ }^{0}\left(\boldsymbol{S}^{2}\right) \mathrm{d} \boldsymbol{S}^{2}=1
$$

on $P_{G}{ }^{0}\left(S^{2}\right)$ in the case that the end-to-end vector $R$ is fixed. Further, introducing $x^{2}=i \eta L / A$, we have from eq $\mathrm{A} 4$ and $\mathrm{A} 5$,

r.h.s. of eq $\mathrm{A} 4=(x / \sin x)^{1 / 2} \exp \left[-\frac{A R^{2}}{L} x \cot x\right]$

r.h.s. of eq $A 5=(\cos x)^{-1 / 2}$

Since it is easy to extend these one-dimensional results to the higher dimensional cases, we can obtain eq 14 and 15.

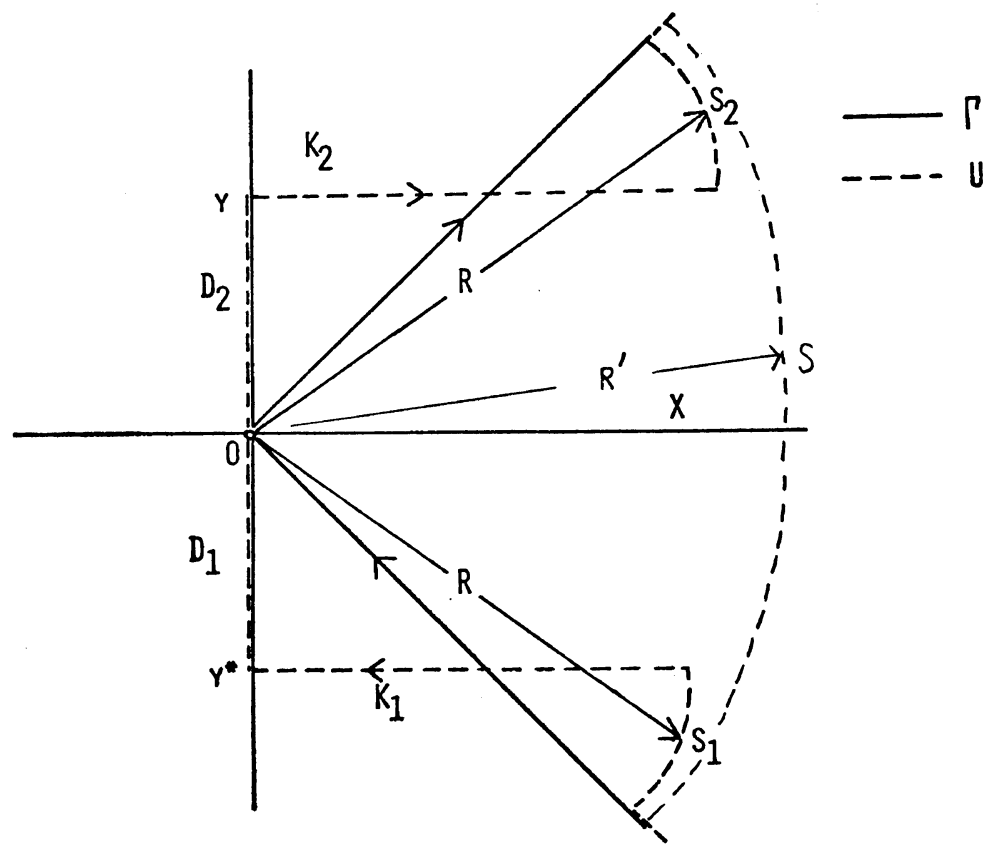

Figure 4. The integration paths chosen for derivations of asymptotic expressions of eq 27 and 28. The path $\Gamma$ is deformed to the path $U$ represented by a dashed line for derivations of eq A19 and 27. The quarter circle $S$ is added to $\Gamma$ for the derivation of eq 28. 


\section{Interaction Effect on Non-Gaussian Distributions}

\section{APPENDIX 2}

Asympotic Solution for Small $\xi$. Equation 53 can be rewritten in two-dimensional space $(d=2)$ in the form

$$
\begin{aligned}
\left\langle\boldsymbol{S}^{2}\right\rangle P_{\mathrm{G}}(\boldsymbol{S})= & \frac{1}{6 i \pi} \int_{\Gamma} \mathrm{d} x\left(x^{2} / \sin x\right) \\
& \times \exp \left[-\xi x^{2} / 6-v( \pm i x)\right]
\end{aligned}
$$

if we take $\Gamma$ as the path of integration in the complex $x$ plane, as shown in Figure 4. As mentioned before, the sign $( \pm)$ means that is taken when $\Gamma$ is in the upper half plane and + is taken when $\Gamma$ is in the lower half plane.

Now when $\xi$ is positive, the path $\Gamma$ can be deformed into the path $U$, which is shown in Figure 4 by a dashed line. The path $U$ consists of two large $\operatorname{arcs} S_{1}$ and $S_{2}$ of radius $R$, two horizontal lines $K_{1}$ and $K_{2}$ from the end point of $S_{1}$ to $y^{*}$ and from $y$ to the end point of $S_{2}$ respectively, where $y$ is a pure imaginary parameter, and two vertical lines $D_{1}$ and $D_{2}$ on the imaginary axis from $y^{*}$ to 0 and from 0 to $y$ respectively, as shown in Figure 4 . It can be shown that the integrals along $D_{1}$ and $D_{2}$ cancel each other and that the integrals along $S_{1}$ and $S_{2}$ tend to zero as the radius, $R$ is increased without limit. Thus eq A9 can be reduced to the form:

$$
\left\langle S^{2}\right\rangle P_{G}\left(S^{2}\right)=\frac{1}{6 \pi i}\left(I_{1}+I_{2}\right)
$$

where

$$
\begin{aligned}
& I_{1}=\int_{K_{1}} \mathrm{~d} x\left(x^{2} / \sin x\right) \exp \left(-\xi x^{2} / 6-i v x\right) \\
& I_{2}=\int_{K_{2}} \mathrm{~d} x\left(x^{2} / \sin x\right) \exp \left(-\xi x^{2} / 6+i v x\right)
\end{aligned}
$$

We consider $I_{2}$. Along the path $K_{2}$, we have $\left|\mathrm{e}^{i x}\right|<\left|\mathrm{e}^{-i x}\right|$, so that the expansion

$$
(\sin x)^{-1}=-2 i \mathrm{e}^{i x} \sum_{n=0}^{\infty} \mathrm{e}^{2 i n x}
$$

holds. Thus $I_{2}$ may be written as

$$
I_{2}=-2 i \sum_{n=0}^{\infty} J_{n}
$$

where $J_{n}$ is given by the equation

$$
J_{n}=\int_{y}^{y+\infty} \mathrm{d} x x^{2} \exp \left[-\xi x^{2} / 6+i(2 n+1) x+i v x\right]
$$

Taking $y=3 i(2 n+1+v) / \xi$, it can be shown that $J_{n}$ is transformed to

$$
\begin{aligned}
J_{n}= & {[3(2 n+1+v) / \xi]^{3} } \\
& \times \exp \left[-3(2 n+1+v)^{2} / 2 \xi\right] \int_{0}^{\infty} \mathrm{d} x(x+i)^{2} \\
& \times \exp \left[-3(2 n+1+v)^{2} x^{2} / 2 \xi\right]
\end{aligned}
$$

Then eq 14 becomes

$$
\begin{aligned}
I_{2}= & -2 i \sum_{n=0}^{\infty}[3(2 n+1+v) / \xi]^{3} \\
& \times \exp \left[-3(2 n+1+v)^{2} / 2 \xi\right] \\
& \times \int_{0}^{\infty} \mathrm{d} x(x+i)^{2} \exp \left[-3(2 n+1+v)^{2} x^{2} / 2 \xi\right]
\end{aligned}
$$

In exactly the same way we obtain

$$
\begin{aligned}
I_{1}= & 2 i \sum_{n=0}^{\infty}[3(2 n+1+v) / \xi]^{3} \\
& \times \exp \left[-3(2 n+1+v)^{2} / 2 \xi\right] \\
& \times \int_{\infty}^{0} \mathrm{~d} x(x-i)^{2} \exp \left[-3(2 n+1+v)^{2} x^{2} / 2 \xi\right]
\end{aligned}
$$

Adding eq A17 and A18 and substituting the result into eq A10, we finally have

$$
\begin{aligned}
\left\langle\boldsymbol{S}^{2}\right\rangle P_{\mathrm{G}}\left(\boldsymbol{S}^{2}\right)= & (6 / \pi)^{1 / 2} \sum_{n=0}^{\infty}\left[3(2 n+1+v)^{2} \xi^{-5 / 2}-\xi^{-3 / 2}\right] \\
& \times \exp \left[-3(2 n+1+v)^{2} / 2 \xi\right] \quad(\mathrm{A} 19)
\end{aligned}
$$

By taking the term $n=0$ only in eq A19, we can obtain eq 54 .

When $\xi$ is negative, the path $\Gamma$ can be deformed into the path consisting of a large arc of radius $R$ from the point on $\Gamma$ in the lower half plane to the point on the negative imaginary axis, i.e., point $-i R$, two vertical lines on the imaginary axis from $-i R$ to 0 and from 0 to $i R$, and a large arc of radius $R$ from $i R$ to the point on $\Gamma$ in the upper half plane. Then, the integral along $\Gamma$ is equal to the integral along the new path, because the domains enclosed by these two paths do not have any singular points of the integrand, and the latter integral goes to zero since the integrals along the vertical lines cancel each other and those along the large arcs tend to zero when $R \rightarrow \infty$

Asympotic Solution for Large $\xi$. If we put $x \rightarrow-x$, the path $\Gamma_{2}$ in the lower half plane changes into the opposite path $\Gamma_{2}^{\prime}$ in the upper 


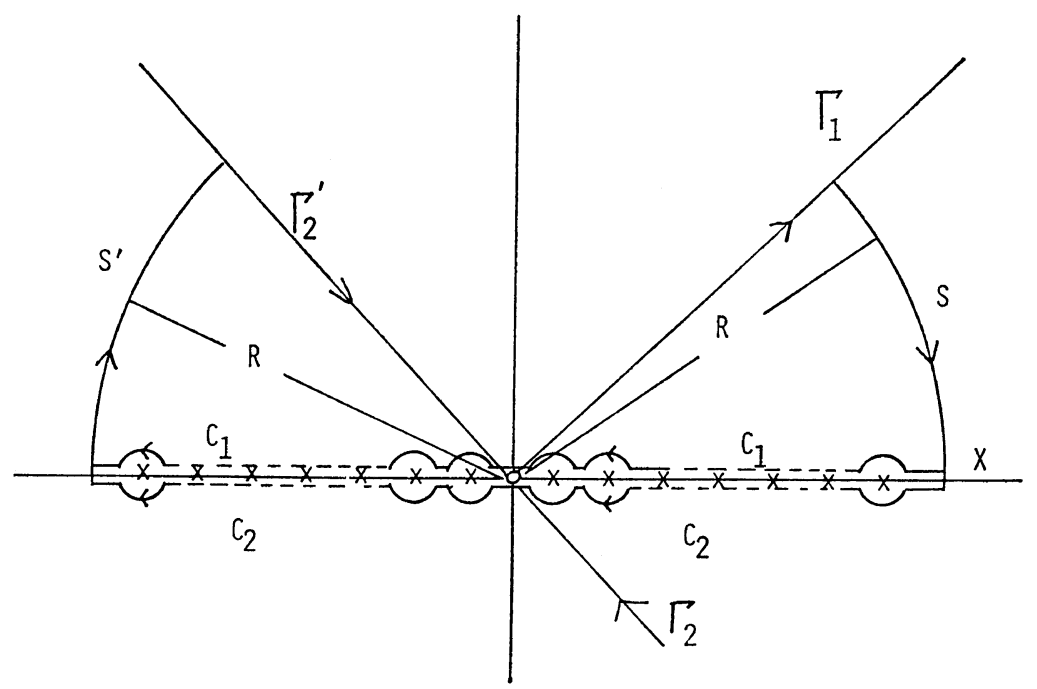

Figure 5. The integration paths chosen for the derivation of the asymptotic expression of eq A23.

half plane, as shown in Figure 5. To the paths $\Gamma_{1}$ and $\Gamma_{2}^{\prime}$ we add two large arcs $S$ and $S^{\prime}$ of radius $R$ and two paths $C_{1}$ and $C_{2}$, which go along the real axis except near the singular points $x_{j}$ and there go away from $x_{j}$ along small semicircles above and below respectively; each $x_{j}$ is a solution of $\sin x=0$ (except $x=0$ ), i.e., $x_{j}=j \pi(j= \pm 1, \pm 2, \pm 3, \cdots)$. The paths are illustrated in Figure 5. Thus, since the function of the integrand has only simple poles at $x_{j}$, the integration along $C_{2} S^{\prime} \Gamma_{2}^{\prime} \Gamma_{1} S$ clockwise is given by the residue theorem as follows:

$-2 \pi i \sum_{j}$ residue at $x_{j}$

$$
\begin{aligned}
= & \int_{\Gamma} \mathrm{d} x\left(x^{2} / \sin x\right) \exp \left[-\xi x^{2} / 6+i v x\right] \\
& -2 i \sum_{j=0}^{\infty} \int_{j \pi+\delta}^{(j+1) \pi-\delta} \mathrm{d} x\left[x^{2} \sin (v x) / \sin x\right] \\
& \times \exp \left(-\xi x^{2} / 6\right)+\Sigma^{\prime}
\end{aligned}
$$

where $\delta$ is infinitesimal positive and $\Sigma^{\prime}$ is the sum of the contributions from along the semicircles. Similarly, the integration along $C_{1} S^{\prime} \Gamma_{2}{ }^{\prime} \Gamma_{1} S$ clockwise, which avoids the poles, is given by

$$
\begin{aligned}
0= & \int_{\Gamma} \mathrm{d} x\left(x^{2} / \sin x\right) \exp \left(-\xi x^{2} / 6+i v x\right) \\
& -2 i \sum_{j=0}^{\infty} \int_{j \pi+\delta}^{(j+1) \pi-\delta} \mathrm{d} x\left[x^{2} \sin (v x) / \sin x\right] \\
& \times \exp \left(-\xi x^{2} / 6\right)+\Sigma^{\prime \prime}
\end{aligned}
$$

By adding eq A20 and A21, and taking into consideration that the contributions of the two arcs $S$ and $S^{\prime}$ and the sum of those of the small circles can be neglected when $\boldsymbol{R} \rightarrow \infty$ and $\delta \rightarrow 0$, respectively, we have the following equation:

$$
\begin{aligned}
\left\langle\boldsymbol{S}^{2}\right\rangle & P_{G}\left(S^{2}\right) \\
= & (1 / 3) \sum_{n=0}^{\infty}(-1)^{n+1} \pi^{2} n^{2} \cos (\pi v n) \\
& \times \exp \left(-\pi^{2} n^{2} \xi / 6\right) \\
& +(1 / 3 \pi) \sum_{j=0}^{\infty} \int_{j \pi+\delta}^{(j+1) \pi-\delta} \mathrm{d} x\left[x^{2} \sin (v x) / \sin x\right] \\
& \times \exp \left(-\xi x^{2} / 6\right)
\end{aligned}
$$

For the second sum of $j$ on the r.h.s. of eq A22, we can neglect all terms except $j=0$ in the limit of $\xi \rightarrow \infty$. Further, expanding $x / \sin x$ in powers of $x$ and extending the integral region $[\delta, \pi-\delta]$ to $[0, \infty]$, we have

$$
\begin{aligned}
\left\langle S^{2}\right\rangle P_{\mathrm{G}} & \left(S^{2}\right) \\
\cong & (1 / 3) \sum_{n=0}^{\infty}(-1)^{n+1} \pi^{2} n^{2} \cos (\pi v n) \\
& \times \exp \left(-\pi^{2} n^{2} \xi / 6\right) \\
& +(1 / 3 \pi) \int_{0}^{\infty} \mathrm{d} x x\left[1+(1 / 6) x^{2}\right. \\
& \left.+(7 / 360) x^{4}\right] \sin (v x) \exp \left(-\xi x^{2} / 6\right)
\end{aligned}
$$

By calculating integrals and taking the term $n=1$ only in eq A 23 we have eq 55 . 


\section{REFERENCES}

1. H. Yamakawa, "Modern Theory of Polymer Solutions," Harper and Row, N. Y., 1971.

2. S. F. Edwards, Proc. Phys. Soc., 85, 613 (1965); S. F. Edwards, ibid., 88, 265 (1966).

3. H. P. Gills and K. F. Freed, Chem. Phys. Lett., 8, 234 (1971).

4. J. Naghizadeh, J. Chem. Phys., 48, 265 (1968).

5. J. des Cloizeaux, Phys. Rev., A, 10, 1665 (1974).

6. D. J. Bruch and M. A. Moore, J. Phys., A, 9, 435 (1976).

7. P. G. de Gennes, Phys. Lett., A, 38, 339 (1973).

8. Z. Alexandrowicz and Y. Accad, J. Chem. Phys., 54, 5338 (1971).

9. N. C. Smith and R. J. Fleming, J. Phys., A, 8, 929; 538 (1975).

10. F. L. MacCrackin, J. Mazur, and C. M. Guttman, Macromolecules, 6, 859 (1973).

11. C. Domb, Adv. Chem. Phys., 15, 229 (1969).

12. C. Domb, J. Chem. Phys., 38, 2957 (1963).

13. M. E. Fisher and B. J. Hiley, ibid., 34, 1253 (1961).

14. T. Minato and A. Hatano, Polymer Preprint, 25, 322 (1976).

15. M. Fixman, J. Chem. Phys., 36, 306 (1962).

16. W. C. Forsman and R. E. Hughes, ibid., 38, 2118 (1963).

17. W. C. Forsman, ibid., 42, 2829 (1965).

18. S. R. Coriell and J. L. Jackson, J. Math. Phys., 8, 1276 (1967).

19. R. F. Hoffman and W. C. Forsman, J. Chem. Phys., 52, 2222 (1970).

20. S. K. Gupta and W. C. Forsman, ibid., 55,
2594 (1971).

21. H. Fujita and T. Norisue, ibid., 52, 1115 (1970).

22. R. Koyama, J. Phys. Soc., 24, 580 (1968).

23. S. K. Gupta and W. C. Forsman, Macromolecules, 5, 779 (1972).

24. N. Wiener, Acta. Math., 55, 117 (1930).

25. R. P. Feynman, Rev. Mod. Phys., 20, 367 (1948).

26. K. F. Freed, Adv. Chem. Phys., 22, 1 (1972).

27. F. W. Weigel, Physics Report, 16C (1975).

28. S. F. Edwards and Y. B. Gulyaev, Proc. Phys. Soc., 83, 495 (1964).

29. M. Saitoh, private communication.

30. T. Minato, Polymer $J$. (to be published).

31. R. P. Feynman, Phys. Rev., 97, 660 (1955).

32. I. C. Sanchez and C. von Frankenberg, J. Chem. Phys., 52, 1617 (1970). These authors confused $Z^{2}$ with the value of the three-dimensional case in their notation. The correct one is $Z^{2}=i N q$ in the two-dimensional case. Refer to 38.

33. P. J. Flory, "Principles of Polymer Chemistry,' Cornell University Press, Ithaca, N. Y., 1953.

34. J. de Cloizeaux, J. Phys., (Paris), 31, 715 (1970).

35. I. M. Gelfand and A. M. Yaglom, J. Math. Phys., 1, 48 (1960).

36. S. G. Brush, Rev. Mod. Phys., 33, 79 (1963).

37. R. P. Feynman and A. R. Hibbs, "Quantum Mechanics and Path Integrals,' McGraw-Hill, N. Y., 1965.

R. P. Feynman, "Statistical Mechanics," Benjamin, N.Y., 1972.

38. T. Minato and A. Hatano, J. Phys. Soc. Jpn. 42, 1992 (1977).

39. K. Solc, Macromolecules, 5, 705 (1972). 